\title{
Molecular genetic characterization reveals linear tumor evolution in a pulmonary sarcomatoid carcinomas patient with a novel PHF20-NTRK1 fusion: a case report
}

\author{
Jianjun $\mathrm{Ge}^{1+}$, Bin $\mathrm{Yao}^{2+}$, Jia Huang ${ }^{2}$, Xue Wu ${ }^{3}$, Hua Bao ${ }^{3}$, Qiuxiang $\mathrm{Ou}^{3}$, Yang W. Shao ${ }^{4,5^{*}}$ and Jun $\mathrm{Chen}^{2^{*+}}$ (D)
}

\begin{abstract}
Background: Pulmonary sarcomatoid carcinoma (SC) consists of both carcinomatous and sarcomatous tumors with high degree of malignancy, rapid progression, and poor prognosis. However, little is known regarding how pulmonary SC develops and progresses.

Case presentation: A 66-year-old male was initially diagnosed with stage Illa lung cancer containing both adenocarcinoma (ADC) and SC. Adjuvant chemotherapy was administrated post-surgery, however, recurrence with SC only soon followed. Mutation profiling of the patient's microdissected ADC and SC components of the primary lesion and recurrent tumor was performed by targeted next-generation sequencing (NGS) of 416 cancer-relevant genes. Our data showed that primary SC/ADC and the recurrent SC shared multiple gene mutations including EGFR, NF1, TP53, CDKN2B, and SMARCA4, while both primary and recurrent SCs had a unique TP53 exon 4 splicing mutation frequently observed in sarcoma. Interestingly, a novel PHF20-NTRK1 fusion was acquired in the recurrent SC, which may be a potential driver for SC recurrence.

Conclusions: The molecular genetic characteristics of tumor tissues at different stages reveals a linear tumor evolution model in this case, and support that the primary SC derived from the original lung ADC during the evolution of the tumor. We also identified a novel PHF2O-NTRK1 fusion, which may contribute to the disease recurrence, and that can be potentially targeted with NTRK1 inhibitors for treatment.
\end{abstract}

Keywords: Lung cancer, Pulmonary sarcomatoid carcinoma, Mutation profiling, Tumor evolution, NTRK1 fusion

\section{Background}

Lung cancer is the most common malignant tumor, of which $87 \%$ is non-small cell lung cancer (NSCLC) [1]. Adenocarcinoma (ADC) is the main histopathological subtype, accounting for more than $50 \%$ of lung cancers. In contrast, sarcomatoid carcinoma (SC), also named collision tumor, occurs with an extreme low incidence of $0.1-1.3 \%$ in all malignant tumors $[2,3]$. It is composed of cancerous and sarcomatoid tumors, and it is still

\footnotetext{
* Correspondence: yang.shao@geneseeq.com; cjcj992@163.com † Jianjun Ge, Bin Yao and Jun Chen contributed equally to this work. ${ }^{4}$ Nanjing Geneseeq Technology Inc., Floor 18, Building B, 3-1 Xinjinhu Road, Pukou District, Nanjing 210032, JS, China

2Department of Radiotherapy and Chemotherapy, Yinzhou People's Hospital, 251 Baizhang E Rd, Jiangdong Qu, Ningbo 315000, Zhejiang, China Full list of author information is available at the end of the article
}

under debate whether the sarcomatoid tumor is differentiated from the cancerous tissue or both tumor types grow in adjacent areas at the same time. SC is featured with a high degree of malignancy, rapid progress, and resistance to conventional radiotherapy or chemotherapy [4]. The elucidation of how it develops and progresses will largely facilitate improving current treatment of SC. Here we report a lung cancer patient diagnosed with both invasive ADC and SC, followed by relapsed SC after surgery. Mutation profiling was performed on the patient's primary and recurrent tumor samples for investigating tumor evolution and genetic alterations contributed to tumor development and progression.

(c) The Author(s). 2019 Open Access This article is distributed under the terms of the Creative Commons Attribution 4.0 International License (http://creativecommons.org/licenses/by/4.0/), which permits unrestricted use, distribution, and reproduction in any medium, provided you give appropriate credit to the original author(s) and the source, provide a link to the Creative Commons license, and indicate if changes were made. The Creative Commons Public Domain Dedication waiver (http://creativecommons.org/publicdomain/zero/1.0/) applies to the data made available in this article, unless otherwise stated. 


\section{Case presentation}

A 66-year-old male with a smoking history of 30 packyear and a drinking history of $60 \mathrm{~g} / \mathrm{d}$ for 30 years visited our hospital following 1 month long coughing symptom with bloody sputum, and was diagnosed with stage IIIa (pT2N2M0) lung cancer on the left lower lobe (Fig. 1a). Thorocoscopic lobectomy was performed immediately to remove the left lower lobe of the lung and related lymph nodes. The excised tumor was confirmed as mixed invasive ADC and SC morphologically and immunohistochemically, accounting for 20 and $80 \%$ of the total tumor content, respectively (Fig. 1a).

We performed mutation profiling of the microdissected ADC and SC compartments of the surgical sample by targeting 416 cancer-relevant genes (GeneseeqOne, Nanjing Geneseeq Technology Inc., China) using hybrid capture-based targeted next-generation sequencing (NGS) on a HiSeq4000 platform (Illumina) [5]. As depicted in Table 1, we observed alterations of multiple oncogenes and tumor suppressor genes that were shared between the two compartments, including EGFR, NF1, SMARCA4, and TP53 mutations, as well as MET gene amplification, consistent with the prior findings that SC has a high mutation rate with the predilection for cooccurrence of more than one driver mutations $[5,8]$. This may account for the high malignancy and aggressive behavior of SC and its poor response to either traditional chemotherapy or radiotherapy as seen in this patient. A rare TP53 deletion (c.97_133 deletion) was detected in both ADC and SC tissues. This variation may result in TP53 exon 4 mis-splicing, which is more frequently seen in sarcoma [9]. Interestingly, an additional TP53 mutation 97-2A $>\mathrm{T}$ that is located right on the splicing accepter of exon 4 was only identified in SC tissue indicating a potential impact of this alteration in SC development, as well as a unique synonymous AXIN2

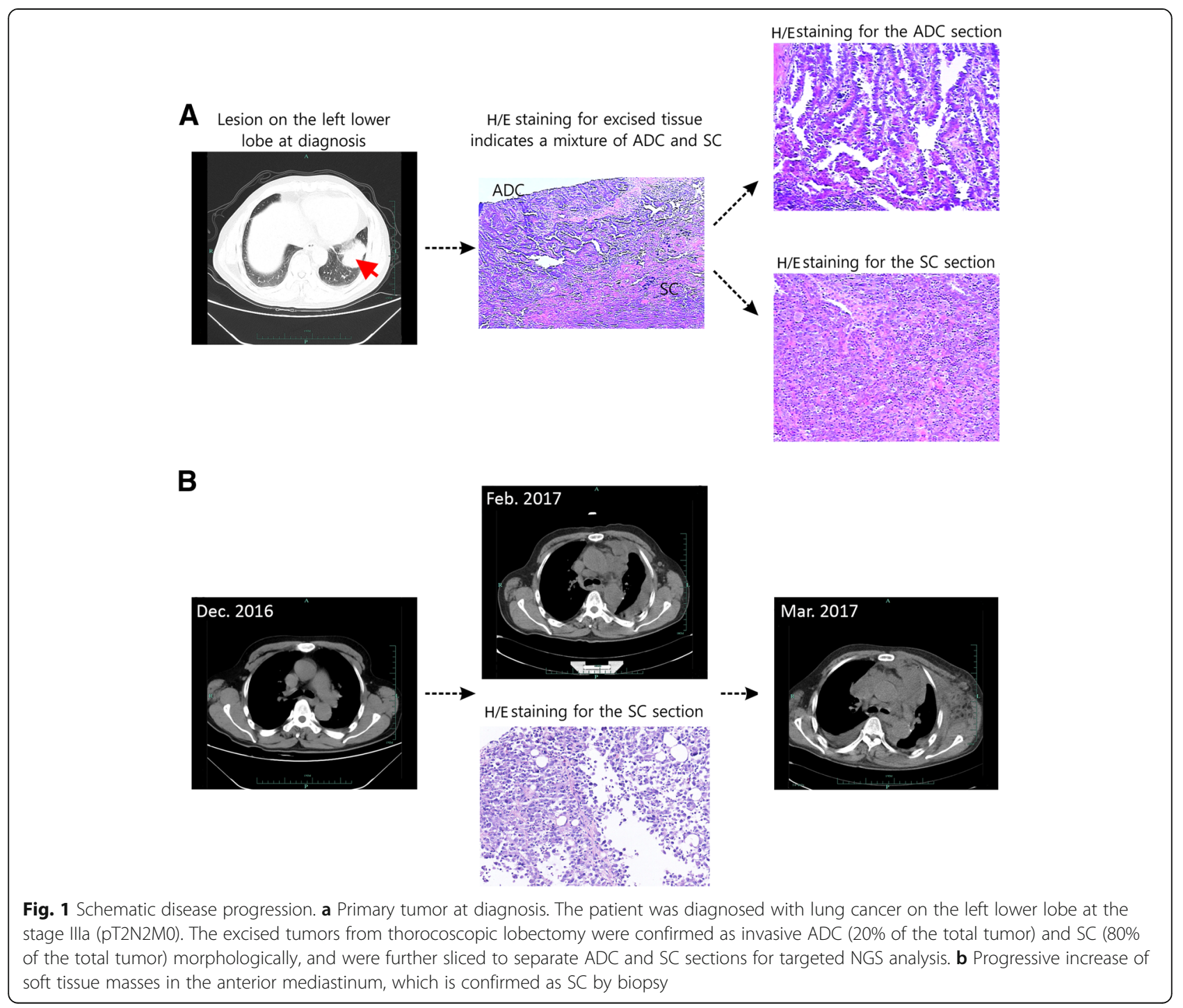


Table 1 Genetic alterations detected in the patient's primary and recurrent tumor. The patient's tumor samples were subject to mutation profiling by targeting a panel of 416 cancer-relevant genes including the introns of 19 genes frequently rearranged in solid tumors, including EGFR, ALK, and NTRKs. The patient's whole blood sample was used to remove the germline mutations. Somatic mutations (filtering criteria: variant allele frequency $>=2 \%$ and $>=5$ supporting reads from both directions) were called for each sample. Genomic fusions were identified by FACTERA [6] using default parameters. Copy number variations (CNVs) were detected using CNVkit [7] with default parameters. n.d., not detected

\begin{tabular}{|c|c|c|c|c|}
\hline Genes & Alternations & Primary ADC & Primary SC & Relapsed SC \\
\hline$\overline{E G F R}$ & p.745_750del & $18.42 \%$ & $13.16 \%$ & $23.90 \%$ \\
\hline NF1 & p.A431S & $26.03 \%$ & $20.63 \%$ & $33.71 \%$ \\
\hline CDKN2B & p.1_7del & $14.29 \%$ & $10.00 \%$ & $17.45 \%$ \\
\hline CDKN2B & p.L34L & $5.80 \%$ & $6.80 \%$ & $8.98 \%$ \\
\hline SMARCA4 & Exon34-35 deletion & $6.4 \%$ & $9.8 \%$ & $15.7 \%$ \\
\hline MET & Amplification & 2.8-fold & 2.6-fold & 4.8-fold \\
\hline TP53 & Exon 4, c.97_133 deletion & $7.69 \%$ & $7.02 \%$ & $14.31 \%$ \\
\hline TP53 & Exon 4 splicing acceptor, 97-2A > T & n.d. & $4.96 \%$ & $10.77 \%$ \\
\hline AXIN2 & p.L128L & n.d. & $8.57 \%$ & $17.05 \%$ \\
\hline PHF2O-NTRK1 & Fusion & n.d. & n.d. & $51.70 \%$ \\
\hline
\end{tabular}

mutation (Table 1). These data suggested a linear evolution model of SC progression from the ADC compartment in the primary tumor of this patient.

About 4 weeks after surgery, adjuvant chemotherapy (carboplatin 0.15 D1-3 + pemetrexed 0.8 D1) was administrated to the patient. However, the patient was diagnosed with cancer relapse within a month. CT scan revealed that soft tissue masses progressively increased in the anterior mediastinum, which was further confirmed as SC by biopsy (Fig. 1b). Genetic characteristics of the recurrent SC was also performed using targeted NGS. Aside from the alterations seen in primary SC tumor tissue, the relapsed SC acquired a novel PHF20NTRK1 fusion where PHF2O intron 2 fused to the intron 4 of NTRK1 at a high variant allele frequency (VAF) (Table 1 and Fig. 2a), resulting in a PHF20-exon 2: NTRK1-exon 5 fusion mRNA with potential in-frame translation (as depicted in Fig. 2b). The resultant fusion protein preserves the whole TRKA kinase domain of NTRK1, and therefore may constitutively activate NTRK1 and contribute to the oncogenesis of the relapsed SC. We further validated the presence of this gene fusion at DNA level in the recurrent SC by PCR amplification of the fusion region followed by Sanger sequencing for sequence confirmation (Fig. 2c). Due to the presence of multiple driver gene alterations, and the unavailability of NTRK1 inhibitor, the patient then received mediastinal tumor palliative radiotherapy (DT $=18 \mathrm{~Gy} /$ $9 \mathrm{~F}$ ), but responded poorly to the treatment and deceased 16 weeks post-operation (Fig. 1b).

\section{Discussion and conclusions}

Pulmonary SC is a rare and aggressive subset of NSCLC with limited treatment options. The understanding of the molecular traits of SC is limited due to the relative rarity of these tumors. Prior studies showed that pulmonary SC tends to have either known driver mutations or high tumor mutation burden [10], and survival probability decreased in patients with mutations detected compared with those without mutations [8]. Point mutations of TP53 and $K R A S$ were most frequently seen in PSC, and KRAS mutations, either alone or in combination with TP53 aberrations, were associated with poor survival [8, 11]. EGFR L858R and G779C mutations have also been reported in PSC $[8,11]$. In this study, EGFR exon19 deletion was detected in both ADC and SC, and we also observed the co-occurrence of multiple mutations of other genes including NF1, TP53, $C D K N 2 B$, and SMARCA4, which may collectively account for the poor response to standard treatments including chemotherapy and radiotherapy, thus resulting in the rapid progression of the disease. Aside from point mutations, we also observed copy number gain of MET in both primary and recurrent tumors, which was shown to be less frequent $(1 / 23,4 \%)$ in PSC [11].

Furthermore, a rare PHF20-NTRK1 fusion was observed in the recurrent SC only. Since mutations such as EGFR exon19 deletion was detected across all samples at VAFs of high confidence established by NGS testing method $[12,13]$, it is unlikely that the absence of the PHF20-NTRK1 fusion was attributed to the low tumor purity of primary tumor samples. Therefore, these lines of evidence corroborate that PHF20-NTRK1 was newly acquired in the recurrent tumor during disease progression. Recently, TRK inhibitors including larotrectinib [14] and entrectinib [15], have been shown to induce clinical meaningful and durable response in patients with NTRK fusion-positive solid tumors. The 

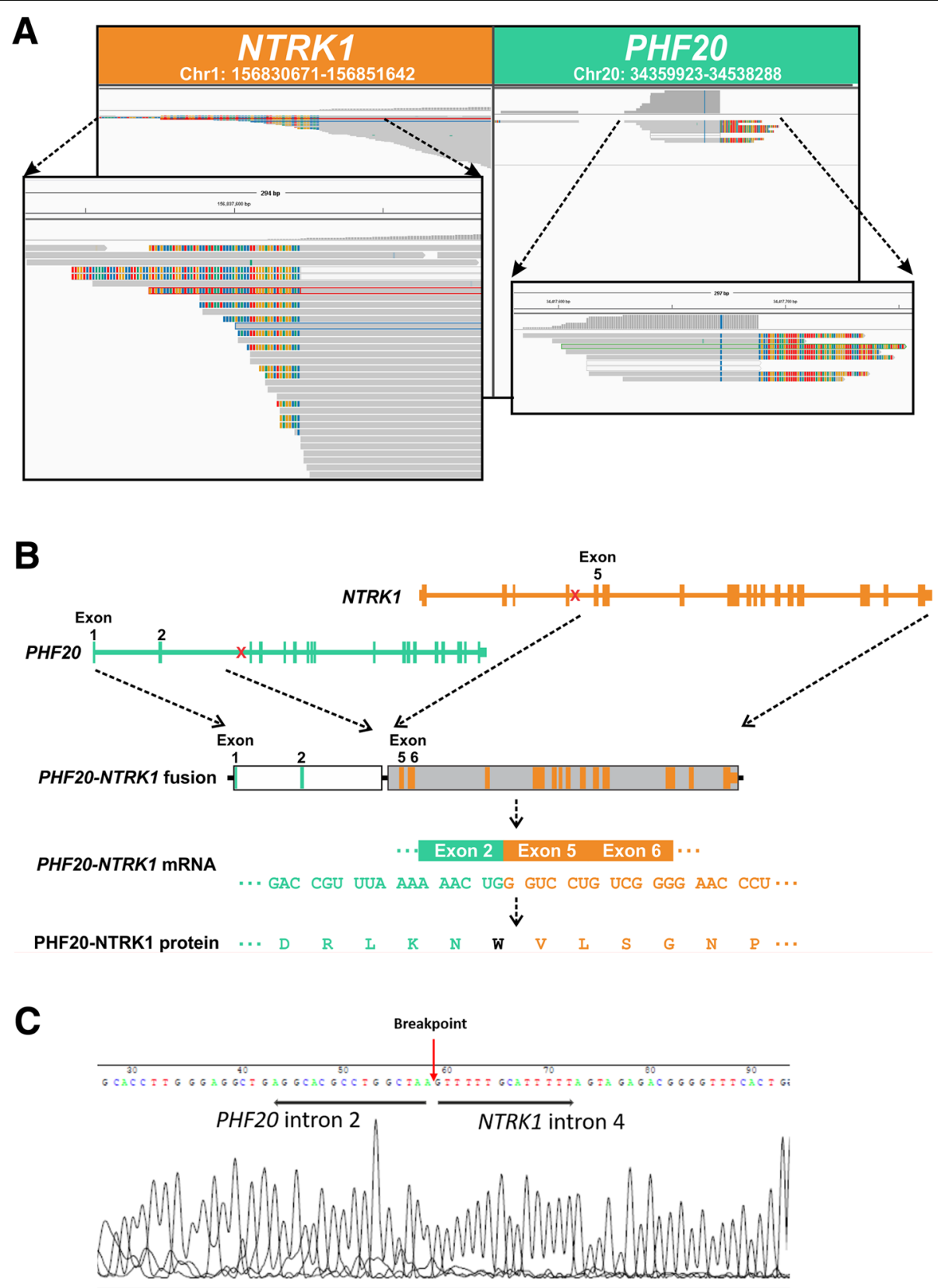

Fig. 2 Novel PHF2O-NTRK1 fusion identified in the relapsed SC tissue. a PHF2O-NTRK1 fusion detected from targeted NGS only in the recurrent SC tissue. $\mathbf{b}$ A diagram depicts the PHF20-NTRK1 fusion at the DNA level, mRNA level and protein level. c The confirmation of the PHF20-NTRK1 fusion by PCR amplification of the fusion region followed by Sanger sequencing

patient could therefore potentially have benefited from those targeted therapies.

Lastly, it remains a mystery how SC coexists with other cancer subtypes, whether both develop from the same cancerous origin or one is evolved from the other. Our study showed that the patient's SC tissue carried all the gene alterations identified in the ADC and two unique alterations including TP53 splicing mutant and a synonymous single nucleic acid variation in AXIN2, suggestive of a linear evolutionary pattern from primary $\mathrm{ADC}$ to SC and further the relapsed SC in the patient.

In summary, mutation profiling of the patient's primary and relapsed tumors revealed a linear tumor evolution model of PSC derived from primary ADC. We also identified a novel PHF20-NTRK1 fusion that may contribute to SC recurrence post-surgery, and the patient could therefore potentially have benefited from targeted therapies against TRKs. 


\section{Abbreviations}

ADC: Adenocarcinoma; NGS: Next-generation sequencing; NSCLC: Non-small cell lung cancer; SC: Sarcomatoid carcinoma; VAF: Variant allele fraction

\section{Acknowledgements}

We would like to thank the patient and his family for giving consent for publication. We also thank all research staff and co-investigators involved in this case study.

\section{Authors' contributions}

$J \mathrm{~J}$ and YWS conceived and designed the study. JG and BY collected the clinical samples, analyzed and interpreted the patient's data. JH performed the histological examination of the tumor tissues. XW and $\mathrm{HB}$ carried out the NGS testing and bioinformatics analysis. JG, BY, YWS and JC co-wrote the manuscript. JC, YWS, QO, and XW contributed to the revision of the manuscript. All authors have read and approved the final manuscript.

\section{Funding}

This research did not receive any specific grant from funding agencies in the public, commercial, or not-for-profit sectors.

\section{Availability of data and materials}

The datasets generated and analyzed during the current study are not publicly available in order to protect the patient's privacy.

\section{Ethics approval and consent to participate}

The patient has given written consent for sample collection, genetic testing, and the use of genetic test data for research purpose. The sample collection and preparation protocols were approved by the Department of Pathology of the Yinzhou People's Hospital, Ningbo, China. Institutional review board (IRB) review and approval was not required for case report.

\section{Consent for publication}

Consent for publication has been obtained from the patient's next of kin stating that the details/images can be available on the Internet and may be seen by the general public.

\section{Competing interests}

Xue Wu, Hua Bao, and Qiuxiang Ou are the employees of Geneseeq Technology Inc.. Yang W. Shao is the employee of Nanjing Geneseeq Technology Inc. Other authors declare that they have no competing interests.

\section{Author details}

'Department of Thoracic Surgery, Yinzhou People's Hospital, Ningbo, Zhejiang, China. ${ }^{2}$ Department of Radiotherapy and Chemotherapy, Yinzhou People's Hospital, 251 Baizhang E Rd, Jiangdong Qu, Ningbo 315000, Zhejiang, China. ${ }^{3}$ Translational Medicine Research Institute, Geneseeq Technology Inc., Toronto, ON, Canada. ${ }^{4}$ Nanjing Geneseeq Technology Inc., Floor 18, Building B, 3-1 Xinjinhu Road, Pukou District, Nanjing 210032, JS, China. ${ }^{5}$ School of Public Health, Nanjing Medical University, Nanjing, JS, China.

Received: 9 February 2018 Accepted: 31 May 2019

Published online: 17 June 2019

\section{References}

1. Miller KD, Siegel RL, Lin CC, Mariotto AB, Kramer JL, Rowland JH, Stein KD, Alteri R, Jemal A. Cancer treatment and survivorship statistics, 2016. CA Cancer J Clin. 2016;66(4):271-89.

2. Goto T, Hirotsu Y, Mochizuki H, Nakagomi T, Oyama T, Amemiya K, Omata M. Stepwise addition of genetic changes correlated with histological change from "well-differentiated" to "sarcomatoid" phenotypes: a case report. BMC Cancer. 2017;17(1):65

3. Spagnolo DV, Heenan PJ. Collision carcinoma at the esophagogastric junction: report of two cases. Cancer. 1980;46(12):2702-8.

4. Ouziane I, Boutayeb S, Mrabti H, Lalya I, Rimani M, Errihani H. Sarcomatoid carcinoma of the lung: a model of resistance of chemotherapy. $\mathrm{N} \mathrm{Am} \mathrm{J}$ Med Sci. 2014;6(7):342-5.

5. Fallet V, Saffroy R, Girard N, Mazieres J, Lantuejoul S, Vieira T, Rouquette I, Thivolet-Bejui F, Ung M, Poulot V, et al. High-throughput somatic mutation profiling in pulmonary sarcomatoid carcinomas using the LungCarta panel: exploring therapeutic targets. Ann Oncol. 2015;26(8):1748-53.

6. Newman AM, Bratman SV, Stehr H, Lee LJ, Liu CL, Diehn M, Alizadeh AA. FACTERA: a practical method for the discovery of genomic rearrangements at breakpoint resolution. Bioinformatics. 2014;30(23):3390-3.

7. Talevich E, Shain AH, Botton T, Bastian BC. CNVkit: genome-wide copy number detection and visualization from targeted DNA sequencing. PLoS Comput Biol. 2016;12(4):e1004873.

8. Lococo F, Gandolfi G, Rossi G, Pinto C, Rapicetta C, Cavazza A, Cesario A, Galeone C, Paci M, Ciarrocchi A. Deep sequencing analysis reveals that KRAS mutation is a marker of poor prognosis in patients with pulmonary Sarcomatoid carcinoma. J Thorac Oncol. 2016;11(8):1282-92.

9. Das P, Kotilingam D, Korchin B, Liu J, Yu D, Lazar AJ, Pollock RE, Lev D. High prevalence of p53 exon 4 mutations in soft tissue sarcoma. Cancer. 2007; 109(11):2323-33.

10. Schrock AB, Li SD, Frampton GM, Suh J, Braun E, Mehra R, Buck SC, Bufill JA, Peled N, Karim NA, et al. Pulmonary Sarcomatoid carcinomas Commonly Harbor either potentially targetable genomic alterations or high tumor mutational burden as observed by comprehensive genomic profiling. J Thorac Oncol. 2017;12(6):932-42.

11. Mehrad M, Roy S, LaFramboise WA, Petrosko P, Miller C, Incharoen P, Dacic S. KRAS mutation is predictive of outcome in patients with pulmonary sarcomatoid carcinoma. Histopathology. 2018;73(2):207-14.

12. Cheng DT, Mitchell TN, Zehir A, Shah RH, Benayed R, Syed A, Chandramohan R, Liu ZY, Won HH, Scott SN, et al. Memorial Sloan Kettering-integrated mutation profiling of actionable Cancer targets (MSKIMPACT): A Hybridization Capture-Based Next-Generation Sequencing Clinical Assay for Solid Tumor Molecular Oncology. J Mol Diagn. 2015;17(3): 251-64.

13. Shu $Y$, Wu X, Tong $X$, Wang $X$, Chang Z, Mao Y, Chen X, Sun J, Wang Z, Hong $Z$, et al. Circulating tumor DNA mutation profiling by targeted next generation sequencing provides guidance for personalized treatments in multiple Cancer types. Sci Rep. 2017;7(1):583.

14. Drilon A, Laetsch TW, Kummar S, DuBois SG, Lassen UN, Demetri GD, Nathenson M, Doebele RC, Farago AF, Pappo AS, et al. Efficacy of Larotrectinib in TRK fusion-positive cancers in adults and children. N Engl J Med. 2018;378(8):731-9.

15. Demetri GD, LP-A AF, Farago SV, Liu SP, Chawla D, Tosi ES, Kim C, Blakely JC, Krauss D, Sigal L, Bazhenova T, John B, Besse J, Wolf T, Seto E, ChowManeval PS, Multani AD, Johnson B, Simmons RC. Doebele: Efficacy and Safety of Entrectinib in Patients with NTRK Fusion-Positive (NTRK-fp) Tumors: Pooled Analysis of STARTRK-2, STARTRK-1 and ALKA-372-001. ESMO 2018 Congress LBA; 2018.

\section{Publisher's Note}

Springer Nature remains neutral with regard to jurisdictional claims in published maps and institutional affiliations.
Ready to submit your research? Choose BMC and benefit from:
- fast, convenient online submission
- thorough peer review by experienced researchers in your field
- rapid publication on acceptance
- support for research data, including large and complex data types
- gold Open Access which fosters wider collaboration and increased citations
- maximum visibility for your research: over $100 \mathrm{M}$ website views per year
At BMC, research is always in progress.
Learn more biomedcentral.com/submissions 